\title{
A simple approach for THE DETERMINATION AND CHARACTERI- ZATION OF TERNARY PHASE DIAGRAMS of aqueous two-phase systems composed of water, poly(ethylene) glycol and sodium carbonate
}

\author{
Emanuel V. Capela, João H.P.M. Santos, Isabel Boal-Palheiros, Pedro J. Carvalho, Joẽo A.P. \\ Coutinho, Sónia P.M. Ventura, and Mara G. Freire \\ University of Aveiro $\bullet$ Aveiro, Portugal
}

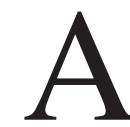

$\mathrm{n}$ important chapter of physical chemistry courses is based on the study of physical transitions that mixtures undergo upon temperature or composition changes. Phase diagrams are an efficient tool to represent this type of information, enabling one to grasp at a glance whether two or more substances are mutually miscible, or how pressure or temperature can be tuned to achieve a particular set of equilibrium conditions. The phase diagrams may be developed using the phase rule, which is, according to Peter Atkins, "the most elegant result of the whole chemical thermodynamics." ${ }^{[1]}$ However mathematically simple, the Gibbs' formulation, i.e. the meaning and the definition of phase and component, may not always be straightforward; for instance, when one or more species undergo a chemical reaction, the number of components may be difficult to establish. Also, the interpretation of phase diagrams, particularly the most complex ones, is not always easy to grasp by students. Beyond their conceptual pedagogical value, phase diagrams bear also a practical interest in the design of separation processes, with wide industrial use. Hence, phase diagrams are an important subject to study and have a crucial role in the academic laboratories and in industry, in the fields of chemical engineering, chemistry and biotechnology.

Aqueous Two-Phase Systems (ATPS) are ternary systems formed when two hydrophilic compounds are dissolved in water above certain concentrations, spontaneously separating into two liquid phases. These systems were proposed by Albertsson ${ }^{[2]}$ and can be formed by polymer/polymer, polymer/salt or salt/salt combinations, dissolved in aqueous media. ${ }^{[3]}$ ATPS have emerged as prominent purification platforms due to the water-richness of the phases, simplicity of preparation, high resolution capacity and ability to be scaled-up. ${ }^{[3]}$ Among the different polymers, poly(ethylene) glycol (PEG) has been widely used in ATPS formulations
Emanuel V. Capela is a Ph.D. student in Chemical Engineering at the University of Aveiro, Portugal. He received his Biochemistry M.S. degree in 2016 from the University of Aveiro, Portugal. His research interests cover the development of new platforms for the purification of biopharmaceuticals using ionic liquids.

João H.P.M. Santos is a Ph.D. student in Chemical Engineering at the University of Aveiro, Portugal, in collaboration with São Paulo University, Brazil. He received his M.S. degree in Industrial and Environmental Biotechnology from the University of Aveiro. His research interests are focused on PEGylation strategies for the production of therapeutic proteins.

Isabel Boal-Palheiros is a lecturer in the Department of Chemistry, University of Aveiro, Portugal. She received her Ph.D. degree in 1997 in Inorganic Chemistry from the University of Aveiro, and she has taught mainly Physical Chemistry subjects. Her current research interests comprise the properties and applications of ionic liquids.

Pedro J. Carvalho is an Assistant Researcher at CICECO, University of Aveiro, Portugal. He received his Ph.D. degree in Chemical Engineering from University of Aveiro in 2011, and from 2012 to 2016 he was a post-doctoral researcher at the same institution. His research focuses on the development of efficient recovery processes in petrochemical refineries, and their modeling.

João A.P. Coutinho is a Full Professor in the Chemistry Department of University of Aveiro, Portugal. He graduated in Chemical Engineering from the University of Porto, Portugal, and received his Ph.D. in Chemical Engineering in 1995 by the Technical University of Denmark. $\mathrm{He}$ is active on the development of sustainable purification processes to be applied in biorefinery.

Sónia P.M. Ventura is an Assistant Researcher at CICECO, University of Aveiro, Portugal. She received her Ph.D. degree in Chemical Engineering from the University of Aveiro in 2011, and was a post-doctoral fellow at the same institution from 2012 to 2014. She works on the Blue Biorefinery concept, developing alternative processes to recover bioactive compounds from marine biomass.

Mara G. Freire is a Coordinator Researcher at CICECO, in the Chemistry Department of University of Aveiro, Portugal, and principal investigator of a European Research Council Grant. She received her Ph.D. degree in Chemical Engineering from the University of Aveiro in 2007. From 2008 to 2013 she was a post-doctoral researcher at ITQB2, New University of Lisbon, Portugal. Her main interests cover the development of sustainable and integrated purification strategies for biopharmaceuticals.

(1) Copyright ChE Division of ASEE 2019 
combined with salts, since it is nontoxic, inexpensive, and biodegradable. ${ }^{[4]}$ Moreover, tuning of ATPS phase diagrams and their characteristics may be achieved by varying the molecular weight of PEG over a considerable range. It is generally observed that the higher the molecular weight of the polymer, the stronger its capability to promote phase separation. ${ }^{[2]}$ On the other hand, and amongst the various salts that can be selected to form ATPS with polymers, sodium carbonate has a strong salting-out capacity and a high ability to promote phase separation. Furthermore, this salt has a diversity of uses, from food additive and acidity regulator to the manufacture of glass, detergents and paper, as well as in brine treatment, water hardness removal and wastewater $\mathrm{pH}$ adjustment. ${ }^{[5,6]}$

In this experimental work the necessary tools are provided for the application of this concept in chemical engineering courses. The determination of ternary phase diagrams of ATPS composed of water, sodium carbonate and PEG of different molecular weights is proposed. Several laboratory experiments aimed at teaching liquid-liquid extraction have been reported, ${ }^{[7-14]}$ yet few involve the determination of the ternary liquid-liquid phase diagrams..$^{[15-19]}$ Since ATPS are here proposed, this work also provides the opportunity to discuss the environmental advantages associated to water-rich systems. Furthermore, the determination of the ternary phase diagrams in this experiment is achieved by simple and inexpensive procedures, that are, nonetheless, the most commonly used methods in the literature for the determination of the solubility (binodal) curves, tie-lines (TLs), tie-line lengths (TLLs), and critical points for these systems. ${ }^{[3]}$

The composition of the system in the saturation curve is gravimetrically determined by weighing the components corresponding to the two-phase formation that is visually detected. A curve is then fitted to those experimental solubility points, which can be used to extrapolate data beyond the experimental range. To determine the tie-lines, a method combining experimental data, mass balance equations and computed data is used. ${ }^{[22]}$ The students need to perform a non-linear regression analysis to solve a series of equations based on the lever rule. Depending on the goals of the course and on the purpose of the lab experiments, the software used by the students to build the phase diagrams may demand no or little programming, or else may challenge their ability to write a program to perform the correlation of the binodal curve and the determination of the tie-lines. For a more advanced training, the liquid-liquid equilibrium data may be modelled by a local composition activity model, such as the NRTL (Non-Random Two Liquid) model. ${ }^{[20]}$ This set of experiments is highly versatile and particularly adequate to be carried out by $3^{\text {rd }}$ or $4^{\text {th }}$ year students in chemical engineering, and also by students in chemistry and biotechnology degrees programs.

The experimentally obtained binodal curve for the ATPS formed by water, $\mathrm{Na}_{2} \mathrm{CO}_{3}$ and $\mathrm{PEG}$ with a molecular weight of $600 \mathrm{~g} / \mathrm{mol}$ (PEG 600) may be represented in a triangular ternary phase diagram, in weight fraction or weight fraction percentages (Figure 1.a). However, to define a ternary system only the amount of two components is needed, the third being dependent; since one of the components is water, the composition of the system may also be represented on an orthogonal diagram (Figure 1.b) highlighting the concentration of the other two components, while the amount of water is omitted. In fact, the use of triangular coordinates

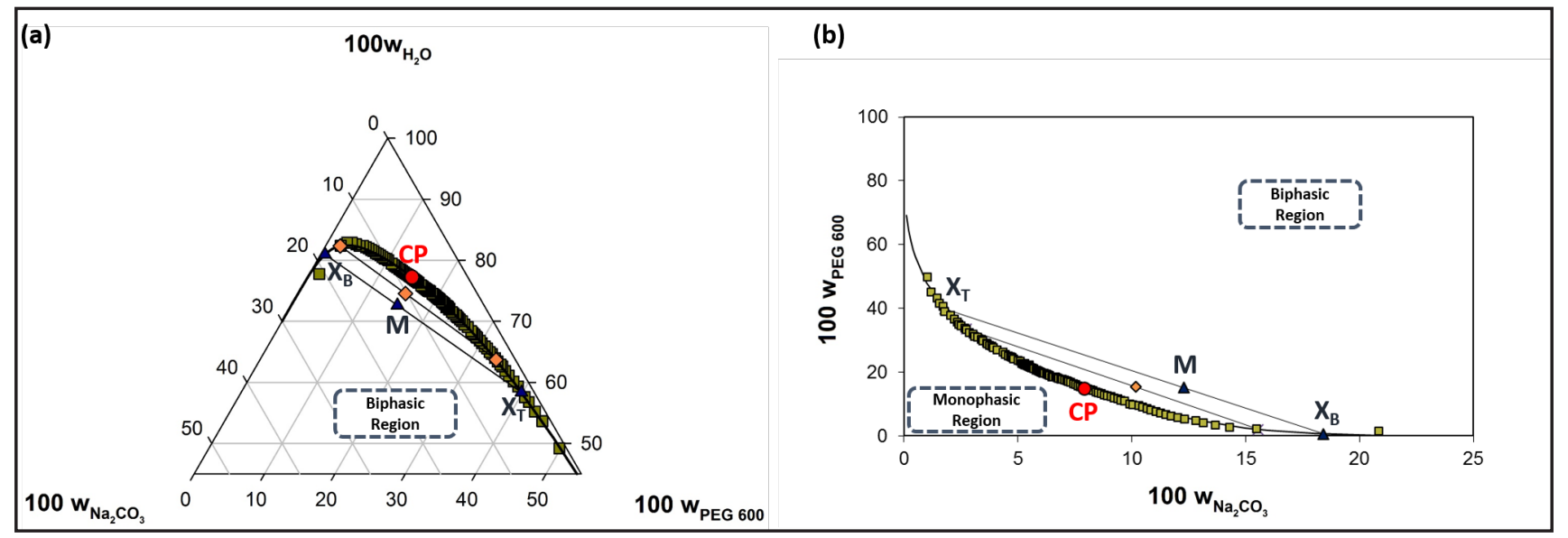

Figure 1. Phase diagrams for the ternary system composed of PEG $600+\mathrm{Na}_{2} \mathrm{CO}_{3}+\mathrm{H}_{2} \mathrm{O}$ at $293 \mathrm{~K}:$ (a) ternary representation (spanning only the composition range of interest) and (b) orthogonal representation. $C P$ represents the critical point of the diagram, $M$ is a random mixture point in the biphasic region of the system, and $X_{T}$ and $X_{B}$ represent the compositions of top and bottom phases of the system, respectively. The labels $100 w_{\mathrm{H}_{2} \mathrm{O}}, 100 \mathrm{w}_{\mathrm{Na} 2 \mathrm{CO} 3}$ and $100 \mathrm{w}_{\mathrm{PEG} 60 \mathrm{O}}$ represent the weight fraction percentage of $\mathrm{H}_{2} \mathrm{O}, \mathrm{Na}_{2} \mathrm{CO}_{3}$ and $\mathrm{PEG} 600$, respectively. 
is not straightforward, and "it may be difficult to estimate the composition represented by a specific point, simply by taking a quick glance at the diagram."[21] The orthogonal representation is the most used approach by researchers in the field of ATPS extractions ${ }^{[3,22,23]}$; thus, it is also important for students to become acquainted with it. It must be remarked that, for high concentration values, the solubility limit of one of the solutes may be reached. In this case, other lines, corresponding to precipitation or solid-liquid or solid-liquid-liquid equilibrium, should be taken into account, but three-phase regions are outside of the scope of this work.

In the phase diagram represented in Figure 1, the biphasic region is localized in the region where the PEG and salt contents are higher, i.e. below the solubility curve in Figure 1.a, and above the solubility curve in Figure 1.b. Each experimental point of the binodal curve corresponds to the phase-forming components concentrations, considering the amount of PEG/salt (whether the direct/indirect method is considered, respectively) in the system (when the cloudy solution appears) and the concentration of all components in the clear system (after water addition). For a given total mixture composition in the biphasic region (M), the mixture undergoes phase separation and forms two coexisting phases, each being represented by the points $\mathrm{X}_{\mathrm{T}}$ and $\mathrm{X}_{\mathrm{B}}$ (compositions of top and bottom phases of the system, respectively), which are the end-points (nodes) of a specific tie-line (TL). Mixtures with total compositions along this specific tie-line have mass or volume ratios that differ in the coexisting phases; yet, the composition of each phase remains the same. The composition difference between these two phases is expressed by the tie-line length (TLL). The length of the tie-lines decreases as they approach the binodal curve and, ultimately, the tie-line length equals zero (TLL=0). At this point, i.e. at the critical point of the ternary system (point $\mathrm{CP}$ ), the compositions of the two coexisting phases become equal - the biphasic system ceases to exist. ${ }^{[24]}$ The TLL is a numerical indicator of how further apart the nodes are and, hence, is a valuable parameter in separation procedures since it correlates trends in the partitioning of solutes between the two phases.

\section{LABORATORY DESCRIPTION}

This laboratory work is divided in two parts: experiment 1 - determination of binodal curves of ternary systems constituted by water, PEGs with molecular weights of 200, 400 and $600 \mathrm{~g} / \mathrm{mol}$ (PEG 200, PEG 400 and PEG 600, respectively) and sodium carbonate $\left(\mathrm{Na}_{2} \mathrm{CO}_{3}\right)$; and experiment 2 - determination of tie-lines, tie-line lengths and critical points of each ATPS and application of the NRTL model to the tie-line data. The students work in groups of two, and a different molecular weight PEG (200, 400 and $600 \mathrm{~g} / \mathrm{mol})$ can be assigned to each group. Two 3-hour periods (classes)

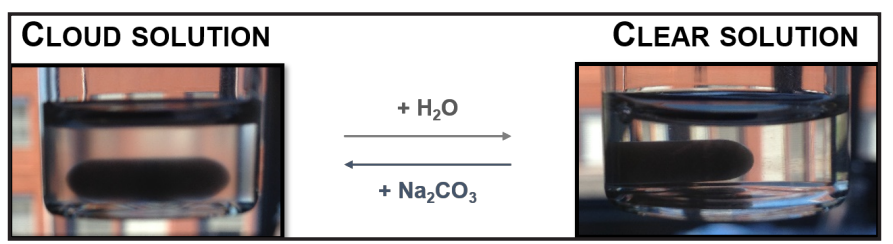

Figure 2. Illustration of the cloud point titration method.

are needed. The first period/class dedicates 30 minutes to discuss/explain the details of the experiment; then, approximately 60 minutes are required to collect experimental data for the first part; a computational period for each group to compute and represent the phase diagram follows; finally, the results obtained for all systems are analyzed and compared. The second period/class comprises 60-70 minutes for the preparation of the several mixtures (at least 5) by each group and the rest of the time is devoted to computational work: determination of mixture compositions, TLs, TLLs and critical points, and application of the NRTL model. The lab equipment requirements for the work are two or more balances $( \pm 0.1 \mathrm{mg})$ (if more than two groups share the same equipment, the time for the experiment to be accomplished increases) and one or two centrifuges (>3000 rpm; 20-50 glass vials).

Experiment 1: Determination of the binodal curves of ternary systems constituted by $\mathrm{PEG}+\mathrm{Na}_{2} \mathrm{CO}_{3}+\mathrm{H}_{2} \mathrm{O}$

The ternary phase diagrams are determined at ambient temperature and pressure by cloud point titration. Stock solutions of sodium carbonate, slightly below the salt solubility saturation in water $\left(100 w_{\text {salt }}=21 \mathrm{wt} \%\right)$, should have been previously prepared to use in the determination of the phase diagrams. Repetitive drop-wise addition of the salt aqueous solution to a PEG aqueous solution $\left(100 w_{P E G}=80\right.$ $\mathrm{wt} \%$ ) previously prepared is carried out until the detection of a cloudy solution, followed by the drop-wise addition of distilled water until the detection of a monophasic region (clear and limpid solution), as illustrated in Figure 2.

To complete the binodal curve, an indirect method is attempted: the repetitive drop-wise addition of the PEG aqueous solution to the salt aqueous solution until the detection of a cloudy solution, followed by the drop-wise addition of distilled water until the detection of a monophasic region (clear and limpid solution). All of these additions are carried out under continuous stirring. The ternary system compositions are determined by weight quantification of all components sequentially added, with the samples always being weighed after identifying each change from cloudy to limpid solution and vice versa.

After the experimental procedure of this first experiment, the students correlate the experimental points of the binodal curve obtained with an equation proposed by Merchuk et $a l .{ }^{[25]}$ which is largely used by researchers in the separations/ extractions field..$^{[3]}$ 


$$
100 w_{P E G}=A \exp \left[B\left(100 w_{\text {salt }}\right)^{35}-C\left(100 w_{\text {salt }}\right)^{3}\right]
$$

where $100 w_{P E G}$ and $100 w_{\text {salt }}$ are, respectively, the PEG and salt weight percentages, and $A, B$ and $C$ are parameters obtained by the regression. This adjustment can be performed using any commercial software provided with non-linear regression and fitting tools; in this work, SigmaPlot ${ }^{\circledR}$ software was used to visualize and plot the curve that best describes the shape and behavior of the data, through the "Curve Fitting and Regression" tool. Although it is merely empiric, the above equation provides a good description of the solubility curve and is particularly useful for "smoothing" experimental deviations or providing regions in the curve where poor or no experimental data exist.

Experiment 2: Determination of tie-lines, tie-line lengths and critical points of each phase diagram

The tie-lines (TLs) are determined by a gravimetric method originally described by Merchuk et al. ${ }^{[25]}$ (at least five TLs are provided for each system, Table 1). The ternary mixtures at the biphasic region described in Table 1 are gravimetrically prepared in centrifugal tubes, by weighing the appropriate amounts of PEG, salt and water, vigorously stirred, and allowed to reach equilibrium by the separation of the two phases through centrifugation for 30 minutes at $3500 \mathrm{rpm}$, at ambient temperature.

After the thermodynamic equilibrium is accomplished and the two-phase formation is visually detected (Figure 3 ), each top and bottom phase is carefully collected using a

\begin{tabular}{|c|c|c|}
\hline \multicolumn{3}{|c|}{$\begin{array}{c}\text { TABLE 1 } \\
\text { Mixture points for each PEG }+\mathrm{Na}_{2} \mathrm{CO}_{3}+ \\
\mathrm{H}_{2} \mathrm{O} \text { system used to find } \mathrm{TLs} \text {. }\end{array}$} \\
\hline \multirow{2}{*}{$\begin{array}{l}\text { PEG } \\
\text { molecular } \\
\text { w eight / } \\
\text { (g/mol) }\end{array}$} & \multicolumn{2}{|c|}{ Mixture Points } \\
\hline & $100 w_{P E G}$ & $100 w_{\text {salt }}$ \\
\hline \multirow{5}{*}{200} & \multirow{5}{*}{30.0} & 8.5 \\
\hline & & 9.0 \\
\hline & & 10.0 \\
\hline & & 11.0 \\
\hline & & 12.0 \\
\hline \multirow{7}{*}{400} & \multirow{7}{*}{25.0} & 8.0 \\
\hline & & 9.0 \\
\hline & & 10.0 \\
\hline & & 11.0 \\
\hline & & 12.0 \\
\hline & & 13.0 \\
\hline & & 14.0 \\
\hline \multirow{5}{*}{600} & \multirow{5}{*}{15.0} & 8.0 \\
\hline & & 10.0 \\
\hline & & 12.0 \\
\hline & & 16.0 \\
\hline & & 18.0 \\
\hline
\end{tabular}

syringe or a Pas-

teur pipette and transferred to a vial, after which the weight of each phase is determined. In the studied ATPS, the top phase is the PEG-rich one.

To determine each TL, the composition of both phases is needed, and this can be carried out using different methodologies. In this work, the composition of each phase is estimated through the application of mass balances to compute the values of four unknowns $\left(100 w_{P E G}^{P E G}, 100 w_{\text {salt }}^{P E G}\right.$, $100 w_{P E G}^{\text {salt }}, 100 w_{\text {salt }}^{\text {salt }}$, which correspond to the percentage weight fraction composition of PEG at the PEGrich phase, the percentage weight fraction composition of salt at the PEG-rich phase, the percentage weight fraction

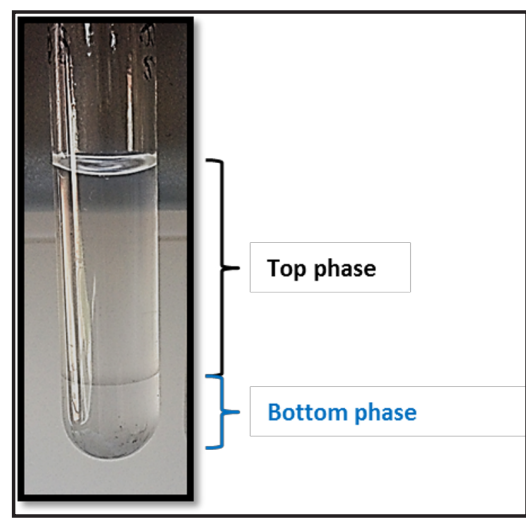

Figure 3. Visual appearance of the two phases formed after the thermodynamic equilibrium is accomplished: top (PEGrich) and bottom (salt-rich) phases. composition of PEG at the salt-rich phase, and the percentage weight fraction composition of salt at the salt-rich phase, respectively) solving the following seven equations system:

$$
\begin{gathered}
100 w_{P E G}^{P E G}=A \exp \left[B\left(100 w_{\text {salt }}^{P E G}\right)^{0.5}-C\left(100 w_{\text {salt }}^{P E G}\right)^{3}\right] \\
100 w_{P E G}^{\text {salt }}=A \exp \left[B\left(100 w_{\text {salt }}^{\text {salt }}\right)^{0.5}-C\left(100 w_{\text {salt }}^{\text {salt }}\right)^{3}\right] \\
100 w_{P E G}^{P E G}=\frac{100 w_{P E G}^{M}}{\alpha}-\frac{1-\alpha}{\alpha}\left(100 w_{P E G}^{\text {salt }}\right) \\
100 w_{\text {salt }}^{P E G}=\frac{100 w_{\text {salt }}^{M}}{\alpha}-\frac{1-\alpha}{\alpha}\left(100 w_{\text {salt }}^{\text {salt }}\right) \\
m_{\text {top }} w_{P E G}^{P E G}+m_{\text {bottom }} w_{P E G}^{\text {salt }}=m_{P E G}^{M} \\
m_{\text {top }} w_{\text {salt }}^{P E G}+m_{\text {bottom }} w_{\text {salt }}^{\text {salt }}=m_{\text {salt }}^{M} \\
\frac{w_{\text {salt }}^{\text {salt }}-w_{\text {salt }}^{M}}{w_{\text {salt }}^{M}-w_{\text {salt }}^{P E E}}=\frac{m_{\text {top }}}{m_{\text {bottom }}}
\end{gathered}
$$

The superscripts PEG and salt designate the PEG-rich (top) and salt-rich (bottom) aqueous phases, respectively, $m$ represents the total weight of each phase, and $M$ is the initial mixture composition. The parameter $\alpha$ is the ratio between the top weight and the total weight of the mixture (experimentally determined). By solving the above system, the concentration values of PEG and of salt in the top and the bottom phases are obtained. This system of seven equations can be solved using any commercial software package that allows the creation and optimization of algorithms to solve this type of systems (e.g. Matlab, Datafitting, NCSS). Our students used Matlab software since this is a software tool they are familiar with. This methodology, which combines 
experimental and computed data, greatly reduces the time to determine the composition of both phases of the tie-lines and has proved to provide reliable results in many ATPS. ${ }^{[3]}$ The other alternative is to analytically determine the composition of each phase for each mixture point.

Once a TL is determined, the calculation of the tie-line length (TLL) is a straightforward procedure, using the equation shown below:

$$
T L L=\sqrt{\left(100 w_{\text {salt }}^{P E G}-w_{\text {salt }}^{\text {salt }}\right)^{2}+\left(100 w_{P E G}^{P E G}-w_{P E G}^{\text {salt }}\right)^{2}}
$$

As mentioned before, several tie-lines are obtained for each system. Also, the compositions of the two phases become equal at the critical point. Consequently, the critical point of each ternary system may be estimated by extrapolation from the compositions of the TLs, using the following linear equation:

$$
100 w_{P E G}^{P E G}=f+g\left(100 w_{\text {salt }}^{\text {salt }}\right)
$$

where $100 w_{P E G}^{P E G}$ and $100 w_{\text {salt }}^{\text {salt }}$ represent the percentage weight fraction compositions of PEG in the PEG-rich phase and $\mathrm{Na}$ ${ }_{2} \mathrm{CO}_{3}$ in the salt-rich phase, respectively, of each determined $\mathrm{TL}$, and $f$ and $g$ are fitting parameters that may be obtained using simple software of calculus and statistics (e.g. Microsoft Excel, SigmaPlot, MatLab).

To provide a thorough understanding of the phase diagram, each group should determine several TLs, the respective TLLs, and the critical point for the selected system, using the experimentally determined binodal data, weight of each phase and the set of equations described above.

In a chemical engineering course, the liquid-liquid equilibrium data determined above can be further modelled using a local composition model, like NRTL (Non-Random Two Liquid). ${ }^{[20]}$ This is an activity coefficient model that correlates activity coefficients in the concerned liquid phase with its mole (or mass) fractions; it accounts for the difference in the interaction energy of a central molecule with molecules of its own kind and that with the molecules of the other kind. This energy difference introduces non-randomness at the local molecular level. Many works have shown the adequacy of using the NRTL model to describe polymer-salt ATPS. ${ }^{[26-28]}$ Although the NRTL model is known to be robust and flexible enough for modeling both the ternary liquid-liquid equilibrium data and the binary subsystems using a common set of parameters, determining this parameter set by fitting the model to ternary experimental tie-lines may lead to wrong descriptions of the binary subsystems (with the sign of the deviation of the ideal mixtures not matching). Thus, in the absence of LLE data for the binary subsystems, the use of the NRTL model relies on reported parameters sets. In this work, the NRTL parameters reported by Sadeghi $^{[29]}$ were adopted, allowing the students to estimate the compositions at each phase through the following equations and objective function $(O F)$,

$$
\begin{gathered}
\left(x_{i}^{P E G}\right)\left(\gamma_{i}^{P E G}\right)=\left(x_{i}^{\text {salt }}\right)\left(\gamma_{i}^{\text {salt }}\right) \\
\ln \left(\gamma_{i}\right)=\frac{\sum_{j} \tau_{j i} G_{j i} x_{j}}{\sum_{k} G_{k i} x_{k}} \\
+\sum_{j}\left[\frac{x_{i} G_{i j}}{\sum_{k} G_{k j} x_{k}}\left(\tau_{i j}-\frac{\sum_{m} \tau_{m j} G_{m j} x_{m}}{\sum_{k} G_{k j} x_{k}}\right)\right] \\
G_{i j}=\exp \left(-\alpha_{i j} \tau_{i j}\right) \\
O F=\sum_{p} \sum_{n} \sum_{k}\left(x_{p, n k}^{2 x p}-x_{p, k}^{\text {calc }}\right)^{2}
\end{gathered}
$$

where $\tau_{i j}$ is the $i$ - $j$ interaction parameters, $\alpha_{i j}$ is related to the non-randomness of the mixture, where the common value of 0.3 is here taken as a default value for all systems.

This set of equations can be solved using any commercial software package that allows the creation and optimization of algorithms (e.g. Matlab, Maple, Mathematica). Our students used Matlab software, since this is a software they are familiar with.

\section{HAZARDS}

Both PEG 200, 400, 600 and sodium carbonate are non-toxic and only slightly hazardous compounds in case of skin contact (irritant, permeator), eye contact (irritant), or inhalation. However, since students always wear laboratory coats, gloves and goggles, risks are minimal.

\section{DATA ANALYSIS}

The systems investigated in this work are formed by water, $\mathrm{Na}_{2} \mathrm{CO}_{3}$ and PEG with three different molecular weights: 200, 400 and $600 \mathrm{~g} / \mathrm{mol}$. The binodal curves for all studied systems are presented below in the conventional triangular form (Figure 4) and orthogonal based form (Figure 5). The experimental weight fraction data of each solubility curve are given in the Supporting Information (available at http:// path.web.ua.pt/publications/SICEE.pdf, Tables SI.1 - SI.3).

When the orthogonal representation is considered (Figure 5), it may be appreciated that the closer the solubility (binodal) curve is to the axes, the larger is the region above the curve and thus the higher is the ability of PEG to suffer a salting-out effect and undergo liquid-liquid demixing in presence of $\mathrm{Na}_{2} \mathrm{CO}_{3}$ in aqueous media. As expected, the influence of the length of the polymer's chains on the phase diagram is notorious. For lower molecular weight polymers, the phase separation occurs at higher concentrations of both the polymer and the salt. In general, the ability of PEG to form ATPS in presence of the salt decreases in the follow- 
ing order: PEG $600>$ PEG $400>$ PEG 200. This behavior is a consequence of the higher hydrophobicity displayed by PEGs of higher molecular weight because they present a lower affinity for water, being thus more easily salted-out by sodium carbonate. Similar trends have been observed in other ATPS composed of polymers and salts described in the literature. ${ }^{[30,31]}$ Salting-out (or salting-in) effects are of high relevance in solution chemistry and have paramount importance in ATPS, and this is a subject that students should be familiarized with

The experimental binodal data for the studied systems were correlated by the empirical relationship described by Equation 1. The regression parameters were estimated by the least-squares regression method, and their values and the corresponding standard deviations are provided in Table SI.4 in the Supporting Information. In general, good correlation coefficients were obtained for all systems, indicating that these fittings can be used to predict data in a region of the phase diagrams where no experimental results are available.

The experimental TLs, along with their respective length (TLL), were determined through the application of Equations 2-9, and are reported in Table SI.5 in the Supporting Information, as well as the TLs compositions correlated by the NRTL model that are reported in Table SI.6. The parameters $f$ and $g$ of Equation 10 were determined for each ternary system and are presented in Table SI.7, as well as the critical point $\left(100 w_{P E G}\right.$ and $\left.100 w_{\text {salt }}\right)$ of each system, resulting from the interception of the linear equation (a result of the extrapolation from the TLs composition) with the binodal curve depicted in Figure 6. The representation of the experimental solubility data, adjusted binodal curve through Equation 1 , TLs and critical point obtained for the system composed of PEG $200+\mathrm{Na}_{2} \mathrm{CO}_{3}+\mathrm{H}_{2} \mathrm{O}$ are shown in Figure 6. The equivalent data for the systems composed of PEG 400 and PEG 600 are presented in the Supporting Information (Figure SI.1 and Figure SI.2, respectively).

\section{STUDENT LEARNING AND FEEDBACK}

This laboratory work was accomplished by 14 students in the $2^{\text {nd }}$ semester of the $3^{\text {rd }}$ year of chemical engineering and biotechnology degrees, as a pilot study before its incorporation in regular classes. The students were given the hand-outs a week before to allow for the necessary preparation, so as to ensure a reasonable understanding of both the experimental tasks and data treatment. Two instructors supervised the lab work to better recognize and identify any difficulties along the way. The students were advised to write down the data in a logbook, but also in the spreadsheet previously prepared by them. Upon completion of both experimental work and data analysis, the students were asked to fill a questionnaire about the experimental work and related concepts. In general, the students were able to carry out both the experimental work and the data treatment with

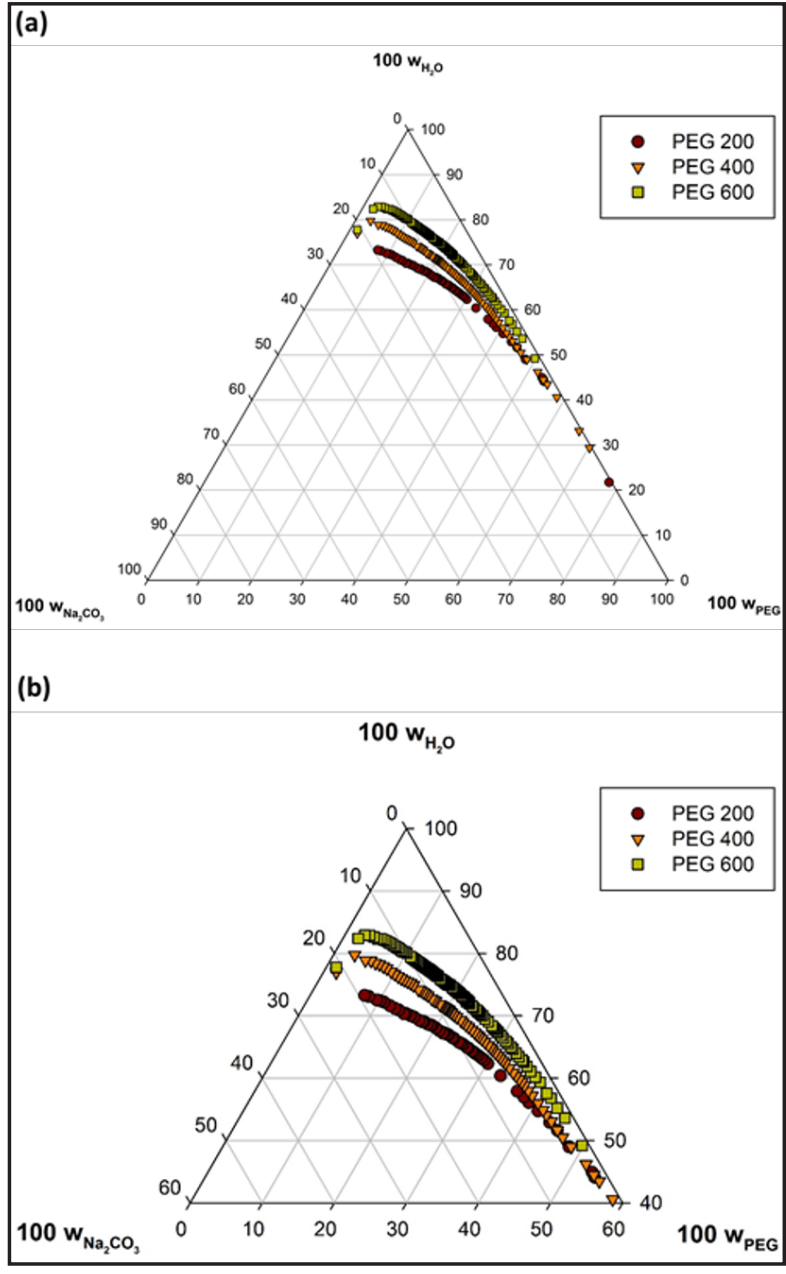

Figure 4. Ternary phase diagram for the systems composed of $\mathrm{PEG}+\mathrm{Na}_{2} \mathrm{CO}_{3}+\mathrm{H}_{2} \mathrm{O}$ at $293( \pm 1) \mathrm{K}:(\bullet) P E G 200 ;(\nabla) P E G$ 400; ( $\square$ ) PEG 600; (a) whole ternary phase diagram and (b) zoom of the phase diagram by changing the axis grade (spanning only part of the composition space of interest).

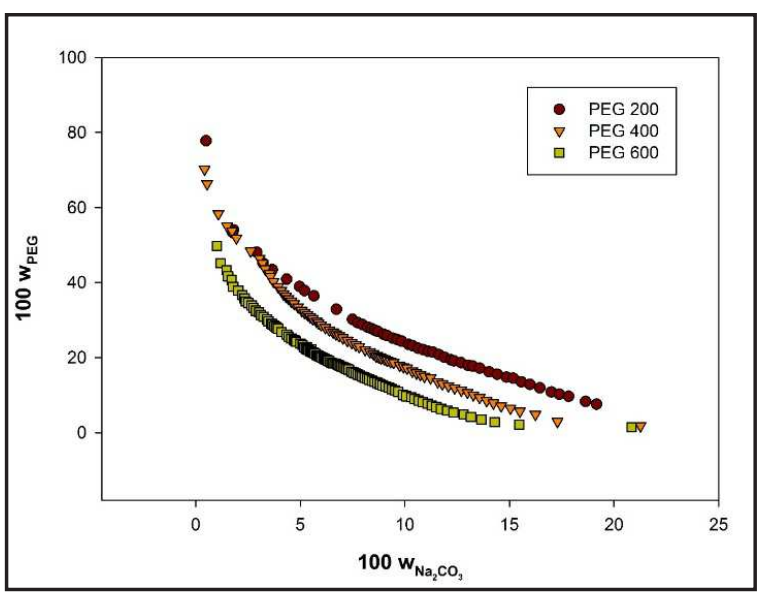

Figure 5. Orthogonal representation of phase diagram for the systems composed of $\mathrm{PEG}+\mathrm{Na}_{2} \mathrm{CO}_{3}+\mathrm{H}_{2} \mathrm{O}$ at $293 \mathrm{~K}$ : (•) PEG 200; ( v) PEG 400; (口) PEG 600 


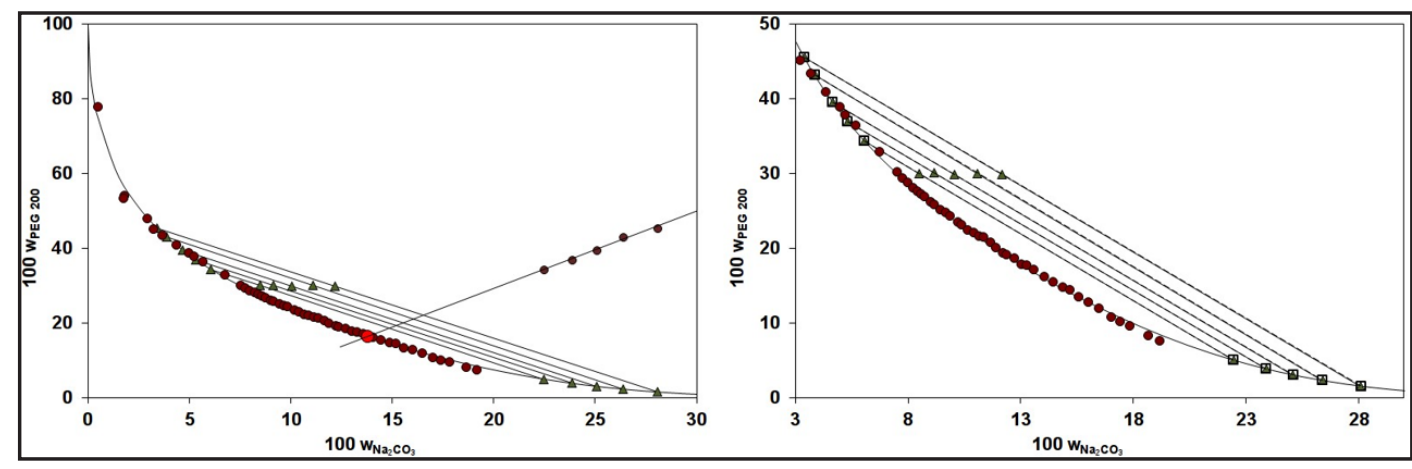

Figure 6. Phase diagram for the ternary system composed of PEG $200+\mathrm{Na}_{2} \mathrm{CO}_{3}+\mathrm{H}_{2} \mathrm{O}$ at $293 \mathrm{~K}$, with the

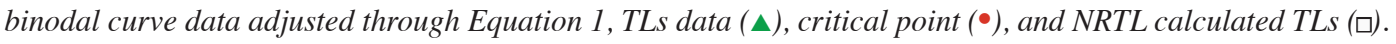

just a few doubts. The global performance of the students according to the evaluated skills is summarized in Table SI.8 in the Supporting Information.

Overall, the students' performance on this laboratory experiment was good or satisfactory in every item under evaluation $(>50 \%)$. All students understood the concept of ternary liquid-liquid phase diagrams, and the experimental determination of the respective binodal curve. They correctly identified the omission of water as the main difference between the orthogonal and the ternary representations of ternary phase diagrams. Although the students were all able to correctly represent the binodal curve data using the orthogonal phase diagrams representation and could identify the mono- and biphasic regions, only $64 \%$ did it correctly for the ternary representation of the phase diagrams. The orthogonal representation turns out to be simpler and more intuitive for non-graduated students. Regarding the molecular mechanisms and salting-out effects associated with the ability to form ATPS, $60 \%$ of the students correlated the effect of the molecular weight of the polymer with its ability to form ATPS, while $80 \%$ had correctly identified the chemical structure of the polymer under study and were aware that it may present different molecular weights.

All students were able to apply Equation (1) to the experimental data, and $80 \%$ pointed out that the main interest of this fitting was the capability of the fitted curve to predict regions of the binodal curve where no experimental data were acquired. This fact reveals that the students felt comfortable with computational tools and understood their relevance. In fact, fitting a curve to experimental data using non-linear regression analysis is not too different from the use of least-squares method to adjust data to a straight line, a procedure they routinely perform. However, when higher order learning skills were at stake, the performance of the students slightly decreases, but is still satisfactory. As data in Table SI.8 in the Supporting Information show, the concept of tie-line length appears to be less clear for the students, since only $64 \%$ of them were able to correctly define and ex- plain it; the concept of critical point caused also some confusion, as some students stated in their feedback. Those are more "abstract" concepts that can be defined but not straightforward visualized. Henceforth, it is important that a discussion/explanation takes place before carrying out the experimental tasks, mainly to ensure that the students understand the principles behind liquid-liquid equilibrium of ternary systems and associated parameters. More than half of the students (57\%) were able to correctly determine the tie-lines, the respective tie-line lengths and the critical points of each phase diagram. Some students felt practical difficulties in the effective separation of the top and the bottom phases, which is probably the major cause for the deviations in their final results. However, this difficulty did not prevent the work to be completed since each group correctly determined at least three TLs. An ineffective separation of top and bottom phases leads to errors on their weight ratio, which may result in crossed TLs; when no parallel TLs are obtained the determination of the critical point of the phase diagrams may be compromised.

\section{DISCUSSION AND CONCLUSIONS}

This laboratory experiment comprises two main sets of experiments: the determination of binodal (solubility) curves of ternary systems constituted by water, PEG (200, 400 and $600 \mathrm{~g} / \mathrm{mol})$ and sodium carbonate $\left(\mathrm{Na}_{2} \mathrm{CO}_{3}\right)$, and the determination of tie-lines, tie-line lengths and critical points for each ternary ATPS. The first task is entirely experimental, while the second requires the combination of experimental data with computer approaches. The experimental procedure used for determining the binodal curves was based on the cloud point titration method, in which the successive endpoints correspond to the appearance and disappearance of turbidity when the system changes from one phase to two phases (or vice versa). The determination of the tie-lines by the combination of experimental and calculated data, thus not requiring the analytical quantification of each component in each phase, allows a substantial reduction in laboratory time and allows the students to get familiarized with the lever rule.

Upon the practical realization of these experiments, students become acquainted with the principles behind ternary liquid-liquid systems and/or ATPS, and their characterization 
by the determination of the binodal curve, tie-lines, tie-line lengths and critical point, as well as in applying the NRTL activity coefficient model to ternary liquid-liquid equilibrium data. They also have the opportunity to understand the influence of using different polymers as phase-forming components and to better address the salting-out effect displayed by inorganic salts.

A relevant feature of this set of experiments is the employment of complementary computational tools to effectively characterize the ternary phase diagrams. The relatively intense use of computational resources, both for calculations and parameters estimation, is a distinct feature from previously proposed experiments and a most valuable one in today's laboratories, both in academic and in industrial environments. The students are encouraged to use commercial spreadsheets that have advanced numerical tools packages, which allow sophisticated calculations in multivariate statistics, as well as linear and nonlinear optimizations that are essential tools in laboratory techniques and scientific research. Non-linear curve fitting is a commonly used tool in chemistry, from deconvolutions of overlapping bands in vibrational or electronic spectra to the analysis of chemical kinetics, and thus it is important for students to become acquainted with these procedures.

Overall, the results showed that this set of lab experiments is a very successful approach in enabling the students to characterize ternary liquid-liquid phase diagrams, as well as to perform the data acquisition and analysis needed. According to the students' preparation and needs, help with the use of software for data treatment may be provided before or along the class; the students' previous acquaintance with the required software is a significant advantage that should be considered.

The students' global overview of the work was positive; they considered the lab class useful in helping them to better understand theoretical concepts related to ternary liquid-liquid phase diagrams. They recognized that putting into practice previously learnt concepts allowed them to fully understand the main parameters needed to establish and characterize a chemical engineering process.

This work may be part of a chemical engineering course

\section{REFERENCES}

1. Atkins P and de Paula J (2002) Atkins' Physical Chemistry, Oxford University Press.

2. Albertsson P-Å (1986) Partition of Cell Particles and Macromolecules: Separation and Purification of Biomolecules, Cell Organelles, Membranes, and Cells in Aqueous Polymer Two-Phase Systems and Their Use in Biochemical Analysis and Biotechnology. John Wiley and Sons, Chichester, UK.

3. Freire MG, Cláudio AFM, Araújo JM, Coutinho JAP, Marrucho IM, Lopes JNC and Rebelo LPN (2012) Aqueous biphasic systems: a boost brought about by using ionic liquids. Chemical Society Reviews, 41 (14): 4966-4995.

4. Chen J, Spear SK, Huddleston JG and Rogers RD (2005) Polyethylene (e.g. $3^{\text {rd }}$ year) or similarly oriented courses where liquid-liquid equilibrium data and separation processes are important. It was designed to be accomplished in two practical laboratory sessions and requires only common laboratory equipment along with modern computational methods to characterize and describe the phase diagrams. Students are encouraged to relate theoretical aspects of phase diagrams and experimental determination and characterization alongside using software to help solving chemical engineering challenges.

This lab work was also meant to update the curriculum to reflect environmental issues for chemical engineering, biotechnology, chemistry and biochemistry students at an intermediate level. The ATPS presented herein will familiarize students with water-rich liquid-liquid systems, which may be used in extraction and separation processes, while avoiding the use of volatile organic solvents.

\section{SUPPORTING INFORMATION}

Additional information is given in the Supporting Information, namely the experimental weight fraction data of each phase diagram; experimental TLs and TLL data; TLs compositions correlated by the NRTL model; fitting parameters and critical points composition; performance and outputs of the students; and individual graphical representation of each phase diagram with the whole characterization. Available at: http://path.web.ua.pt/publications/SICEE.pdf.

\section{ACKNOWLEDGEMENTS}

This work was developed within the scope of the project CICECO-Aveiro Institute of Materials, POCI-01-0145-FEDER-007679 (FCT Ref. UID/CTM/50011/2013), financed by national funds through the FCT/MEC and when appropriate co-financed by FEDER under the PT2020 Partnership Agreement. E.V. Capela, J.H.P.M. Santos, S.P.M. Ventura and P.J. Carvalho acknowledge FCT for the $\mathrm{PhD}$ grants SFRH/BD/126202/2016 and SFRH/BD/102915/2014, and the IF contracts IF/00402/2015 and IF/00758/2015, respectively. M.G. Freire acknowledges the European Research Council (ERC) for the Starting Grant ERC-2013- StG337753.

glycol and solutions of polyethylene glycol as green reaction media. Green Chemistry, 7 (2): 64-82.

5. Marques CF, Mourão T, Neves CM, Lima ÁS, Boal-Palheiros I, Coutinho JAP and Freire MG (2013) Aqueous biphasic systems composed of ionic liquids and sodium carbonate as enhanced routes for the extraction of tetracycline. Biotechnology Progress, 29 (3): 645-654.

6. Block C (2003) Human and environmental risk assessment on ingredients of household cleaning products. Jornadas - Comite Espanol de la Detergencia, 33: 39-44.

7. Turner DE (1994) An experiment to demonstrate the effect of $\mathrm{pH}$ on partition coefficients in liquid-liquid extraction. Journal of Chemical Education, 71 (2): 173. 
8. Loyo-Rosales JE, Torrents A, Rosales-Rivera GC and Rice CP (2006) Linking laboratory experiences to the real world: the extraction of octylphenoxyacetic acid from water. Journal of Chemical Education, 83 (2): 248

9. Horowitz G (2003) A discovery approach to three organic laboratory techniques: extraction, recrystallization, and distillation. Journal of Chemical Education, 80 (9): 1039.

10. Jones MM and Champion GR (1978) Simple liquid-liquid extraction experiment for freshmen. Journal of Chemical Education, 55 (2): 119.

11. Radford SA, Hunter Jr RE, Barr DB and Ryan PB (2013) Liquid-liquid extraction of insecticides from juice: an analytical chemistry laboratory experiment. Journal of Chemical Education, 90 (4): 483-486.

12. Revell KD (2011) Separation of the components of a commercial analgesic tablet: a two-week sequence comparing purification by two-base extraction and column chromatography. Journal of Chemical Education, 88 (10): 1413-1415.

13. Wigman LS and Kelsch CT (1992) Separation science and chromatography. A colorful introduction. Journal of Chemical Education, 69 (12): 991.

14. Gordon P, Pezhathinal AJ, Rocke K, Susanto L, Handke D, Chan-YuKing R, Losey EN and Keiter RL (2006) Colorful chemical demonstrations on the extraction of anionic species from water into ether mediated by tricaprylylmethylammonium chloride (Aliquat 336), a liquid-liquid phase-transfer agent. Journal of Chemical Education, 83 (8): 1161.

15. Clarke J (1974) Tie lines in phase diagrams for ternary liquid systems. Journal of Chemical Education, 51 (4): 255.

16. Clare B, Hefter G and Kloeden P (1985) The representation of ternary liquid composition diagrams. Journal of Chemical Education, 62 (8): 680 .

17. Stead RJ and Stead K (1990) Phase diagrams for ternary liquid systems. Journal of Chemical Education, 67 (5): 385.

18. Udale BA and Wells JD (1995) A ternary phase diagram for a less hazardous system. Journal of Chemical Education, 72 (12): 1106.

19. Francis AW and Smith NO (1969) Ternary liquid systems. Journal of Chemical Education, 46 (12): 815.

20. Renon H and Prausnitz J (1969) Estimation of parameters for the NRTL equation for excess Gibbs energies of strongly nonideal liquid mixtures. Industrial \& Engineering Chemistry Process Design and Development, 8 (3): 413-419.
21. MacCarthy P (1983) Ternary and quaternary composition diagrams: an overview of the subject. Journal of Chemical Education, 60 (11): 922.

22. Capela EV, Quental MV, Domingues P, Coutinho JAP and Freire MG (2017) Effective separation of aromatic and aliphatic amino acid mixtures using ionic-liquid-based aqueous biphasic systems. Green Chemistry, 19 (8): 1850-1854.

23. Taha M, Quental MV, e Silva FA, Capela EV, Freire MG, Ventura SP and Coutinho JAP (2017) Good's buffer ionic liquids as relevant phase-forming components of self-buffered aqueous biphasic systems. Journal of Chemical Technology \& Biotechnology, 92 (9): 2287-2299.

24. Kaul A (2000) The phase diagram, in Aqueous Two-Phase Systems: Methods and Protocols. Hatti-Kaul R (Ed.), Springer, Totowa, New Jersey, 11-21.

25. Merchuk JC, Andrews BA and Asenjo JA (1998) Aqueous two-phase systems for protein separation: Studies on phase inversion. Journal of Chromatography B: Biomedical Sciences and Applications, 711 (1): 285-293.

26. Zafarani-Moattar MT and Sadeghi R (2002) Measurement and correlation of liquid-liquid equilibria of the aqueous two-phase system polyvinylpyrrolidone-sodium dihydrogen phosphate. Fluid Phase Equilibria, 203 (1-2): 177-191.

27. Zafarani-Moattar MT and Hamzehzadeh S (2005) Liquid-liquid equilibria of aqueous two-phase systems containing polyethylene glycol and sodium succinate or sodium formate. Calphad, 29 (1): 1-6.

28. Sadeghi R (2005) Measurement and correlation of phase equilibria for several PVP+ salt aqueous two-phase systems at 303.15 K. Fluid Phase Equilibria, 237 (1-2): 40-47.

29. Sadeghi R (2006) A modified segment-based nonrandom two-liquid model for the calculation of vapor-liquid equilibrium of aqueous polymer-salt solutions. Chemical Engineering Science, 61 (23): 7786-7794

30. Azevedo AM, Gomes AG, Rosa PA, Ferreira IF, Pisco AM and Aires-Barros MR (2009) Partitioning of human antibodies in polyethylene glycol-sodium citrate aqueous two-phase systems. Separation and Purification Technology, 65 (1): 14-21.

31. de Souza RL, Campos VC, Ventura SP, Soares CM, Coutinho JAP and Lima ÁS (2014) Effect of ionic liquids as adjuvants on PEG-based ABS formation and the extraction of two probe dyes. Fluid Phase Equilibria, 375: $30-36$. $\square$ 